\title{
Dichromate effect on the passive layer of 316L stainless steel
}

\author{
M. Askarian 1 , M. Peikari ${ }^{2}$ \& S. Javadpour ${ }^{3}$ \\ ${ }^{I}$ Pars Oil and Gas Company, Iran \\ ${ }^{2}$ Petroleum University of Technology, Iran \\ ${ }^{3}$ Shiraz University, Iran
}

\begin{abstract}
The resistance of a passive layer of stainless steel depends on the alloy composition and conditions in which it is generated. Chemical treatment on steel parts could improve the passive layer. In present work, the effect of passivation treatment, with immersion in a dichromate solution with concentrations 10, 20, and $40 \mathrm{~g} / \mathrm{l}$ for 24 and $72 \mathrm{hr}$ on the stainless steel 316L was investigated. Polarization and electrochemical impedance spectroscopy studies, conducted in $\mathrm{NaCl} 3.5 \%$, show that $20 \mathrm{~g} / \mathrm{l}$ dichromate passivation treatment for $72 \mathrm{hr}$ would improve corrosion resistance markedly due to the diffusion control reaction. SEM results imply that passivation treatment is advantageous to improve the smoothness. XPS analysis indicated that a stable mixture compound layer was formed and the passive film can be described by a bi-layer model (inner oxide and outer hydroxide layers).
\end{abstract}

Keywords: passive layer, $316 \mathrm{~L}$ stainless steel, dichromate, chemical treatment, EIS, SEM, XPS.

\section{Introduction}

The high corrosion resistance of stainless steels is primarily due to the oxide layers formed in its surfaces, and for this reason passivation is a question of considerable technical and economical importance [1]. The protective film is normally an oxide of the metal with oxygen dissolved in the contacting solution or by an oxidizing material [4]. The resistance of the passive films is determined by the alloy composition, medium and conditions in which it is generated, causing the passivation of stainless steels to be a very complex process, not still 
completely understood [1]. The presence of exogenous surface contamination, including dirt, grease, free iron from contact with steel tooling, and so forth, may interfere with the formation of the passive film. The cleaning of these contaminants from the stainless steel surface will facilitate the spontaneous passivation by allowing the oxygen uniform access to the surface. The passive film may be augmented by chemical treatments that provide an oxidizing environment for the stainless steel surface [5]. This redox system could be any oxidizing agent that forms an oxide layer on steel, such as ferric, nitrite or chromate ions $[4,6]$. Stainless steel parts shall be treated in specified aqueous solutions and maintained within the specified temperature range for the specified time.

The purpose of this study is to introduce chemical treatment to enhance corrosion resistance of $316 \mathrm{~L}$ stainless steel parts.

\section{Experiment}

Specimens used in this study were $316 \mathrm{~L}$ stainless steel. The composition in $\mathrm{wt} \%$ was $0.021 \% \mathrm{C}, 17.61 \% \mathrm{Cr}, 12.45 \% \mathrm{Ni}, 2.29 \% \mathrm{Mo}, 0.069 \% \mathrm{Si}, 1.05 \% \mathrm{Mn}$, $0.020 \% \mathrm{~S}, 0.031 \% \mathrm{P}$, and bal. Fe. They were mounted with an exposed area to $1 \times 1 \mathrm{~cm}^{2}$. The surface was mechanically polished by 220 grit $\mathrm{SiC}$ paper followed with 320/400/800/1000 grit paper to have a comparable surface roughness. Then they were rinsed with deionized water. Passivation treatments were immersed according to the specification in table 1.

Table 1: $\quad$ Specification of dichromate-passivation treatments.

\begin{tabular}{|c|c|c|}
\hline Sample & Concentration $(\mathrm{g} / \mathrm{l})$ & Time $(\mathrm{hr})$ \\
\hline 1 & 10 & 24 \\
\hline 2 & 20 & 24 \\
\hline 3 & 40 & 24 \\
\hline 4 & 10 & 72 \\
\hline 5 & 20 & 72 \\
\hline 6 & 40 & 72 \\
\hline
\end{tabular}

Attention shall be given to maintaining adequate volume, concentration, purity, and temperature control appropriate to the size of the stainless steel specimens to be treated.

Immediately after removal from the passivating solution the specimens were thoroughly rinsed, using spray washes [5].

The original specimen was tested as-received, without passivation treatment as reference and for comparative purposes. The corrosion properties and composition of the passive layer were studied using DC polarization and electrochemical impedance spectroscopy (EIS). Electrochemical tests were conducted using potentiostat, Model Zahner, in $3.5 \% \mathrm{NaCl}$ solution at room temperature opening air. Saturated calomel electrode (SCE) was used as a reference electrode and platinum as an auxiliary electrode. Potentiodynamic 
polarization curves were plotted from $-200 \mathrm{mV}_{\mathrm{SCE}}$ to $+200 \mathrm{mV}_{\mathrm{SCE}}$ at a polarization scan rate of $0.1 \mathrm{mV} / \mathrm{s}$; EIS measurements were performed in the frequency range from $100 \mathrm{mHz}$ to $100 \mathrm{kHz}$ with a logarithmic sweeping frequency of 4 steps/decade. EIS involved the imposition of a $10 \mathrm{mV}$ amplitude sine-wave and the measurements were carried out at the $\mathrm{E}_{\text {corr. }}$.

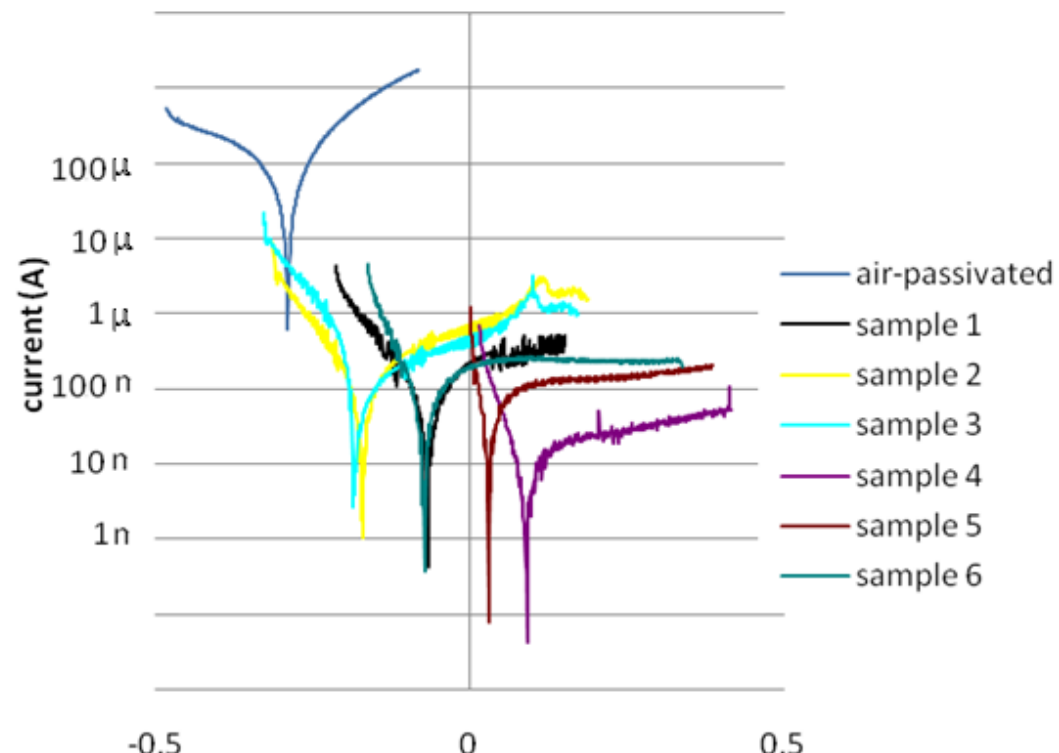

Potential(V)

Figure 1: $\quad$ Polarization curves of specimens.

The electrochemical results for chromium-passivated specimen were compared with air-passivated indicated which passivation treatment gives superior corrosion resistance. Then the surfaces, microstructure and composition of that specimen were investigated using scanning electron microscopy (SEM), and X-ray photoelectron spectroscopy (XPS) to determine cause for this behaviour. SEM was performed using JEOL JXA-840 equipment at the voltage $(15 \mathrm{kV})$. The XPS spectra were obtained with a VG Microtech electron spectrometer, model MT 500, using an $\mathrm{Mg} \mathrm{K} \alpha_{1.2}$ anode $\mathrm{X}$-ray source $(\mathrm{h} v=1253.6$ $\mathrm{eV}$ ) with a primary beam energy of $15 \mathrm{kV}$ and an electron current of $20 \mathrm{~mA}$. The pressure in the analysis cabinet was maintained at $1 \times 10^{-9}$ Torr throughout the measurements. The regions of interest were $\mathrm{O}_{1 \mathrm{~s}}, \mathrm{Cr}_{2 \mathrm{p}}$, and $\mathrm{Fe}_{2 \mathrm{P}}$. The concentration of each element was estimated by measuring the respective peak area in the high-resolution XPS spectrum and using elemental sensitivity factors. The specimens were analyzed by means of argon-ion sputtering on the original surface and after 30, 90 and $390 \mathrm{~s}$ argon-ion sputtering was carried out with primary beam energy of $5 \mathrm{kV}$ and an ion density of $1 \mu \mathrm{A} / \mathrm{cm}^{2}$, recording the corresponding XPS spectrum for each sputtering cycle. 


\section{Results and discussion}

\subsection{Electrochemical}

Fig. 1 shows polarisation curves for the chromium-passivated and air-passivated specimens tested in a $3.5 \% \mathrm{NaCl}$ solution. The $\mathrm{E}_{\text {corr }}$ and $\mathrm{I}_{\text {corr }}$ of each specimen are mentioned in table 2 [2].

Figure 2 presents Nyquist plots for chromium-passivated and air-passivated specimens tested in a $3.5 \% \mathrm{NaCl}$ solution.

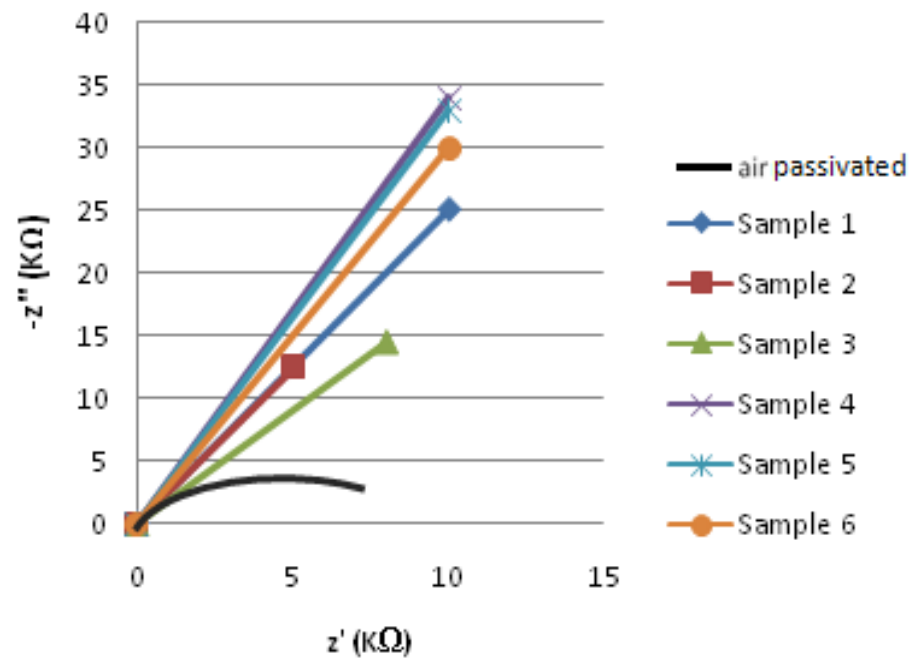

Figure 2: $\quad$ Nyquist plot of samples 1-6.

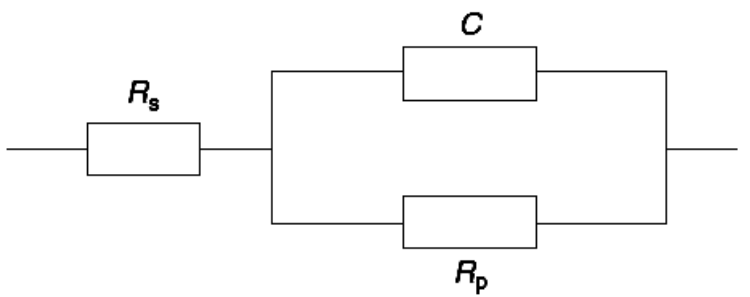

Figure 3: $\quad$ Randles Simple equivalent circuit of the air-passivated sample.

EIS curves were interpreted by an equivalent circuit. Fig.3 shows Randles Simple equivalent circuit of air-passivated specimen. In this equivalent circuit $R_{p}$ is the polarization resistance of the outer surface of the working electrode, $C$ the capacity of the electrochemical double layer of the outer surface of the working electrode and $\mathrm{R}_{\mathrm{s}}$ the ohmic resistance between the reference electrode and the outer surface of the working electrode $[7,8]$.

The EIS curve of chromium-passivated specimen is more complicated. Imaginary impedances do not increase and capacitive semicircles do not 
complete. This behaviour indicates reaction is under diffusion control. Therefore the Warburg diffusion impedance is an inevitable element in circuit. Also capacitive is replaced with $\mathrm{CPE}$ (constant phase element) to fit with experimental curve $[7,11]$.

Table 2 indicates $R_{p}$ obtained from equivalent circuit of each specimen. Electrochemical tests results show chromium treatment improves corrosion resistance of stainless steel. Increasing concentration of chromium and duration of immersion generally has more improving effect. However, too concentrated solution leads to a decrease in corrosion resistance relatively due to transfer into transpassive region $[2,7]$.

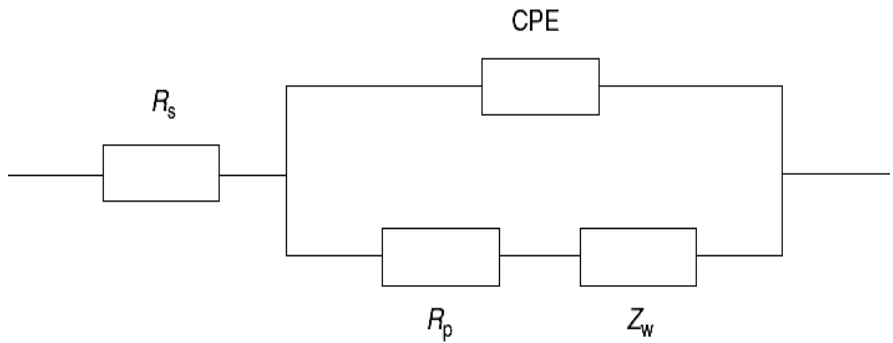

Figure 4: $\quad$ Equivalent circuit of the chromium-passivated samples.

Table 2: $\quad$ EIS and polarization tests results.

\begin{tabular}{|c|c|c|c|}
\hline sample & $(\mathrm{mV})$ Corrosion potential & $\begin{array}{c}\text { Corrosion rate } \\
\left(\mathrm{nA} / \mathrm{cm}^{2}\right)\end{array}$ & $\begin{array}{c}\text { Resistance } \\
\left(\Omega / \mathrm{cm}^{2}\right)\end{array}$ \\
\hline Air-passivated & -289 & 5100 & $1.2 \times 10^{3}$ \\
\hline 1 & -68 & 75 & $2 \times 10^{5}$ \\
\hline 2 & -170 & 94 & $3.67 \times 10^{5}$ \\
\hline 3 & -157 & 274 & $8.8 \times 10^{4}$ \\
\hline 4 & 87 & 10 & $4.5 \times 10^{5}$ \\
\hline 5 & 29 & 6 & $5.5 \times 10^{5}$ \\
\hline 6 & -72 & 59 & $1.3 \times 10^{5}$ \\
\hline
\end{tabular}

\subsection{SEM}

No visual difference was seen among the seven samples, but SEM examination indicated some slight topography changes. Figs. 5 and 6 show SEM for airpassivated and the best passivated specimen (sample No.5) respectively. The passivation treatment is advantageous to improve the smoothness [3].

\subsection{XPS}

XPS analysis of stainless steel surfaces treated by various chemicals can provide valuable information about passivation layer composition, and its ability to protect against corrosion. 


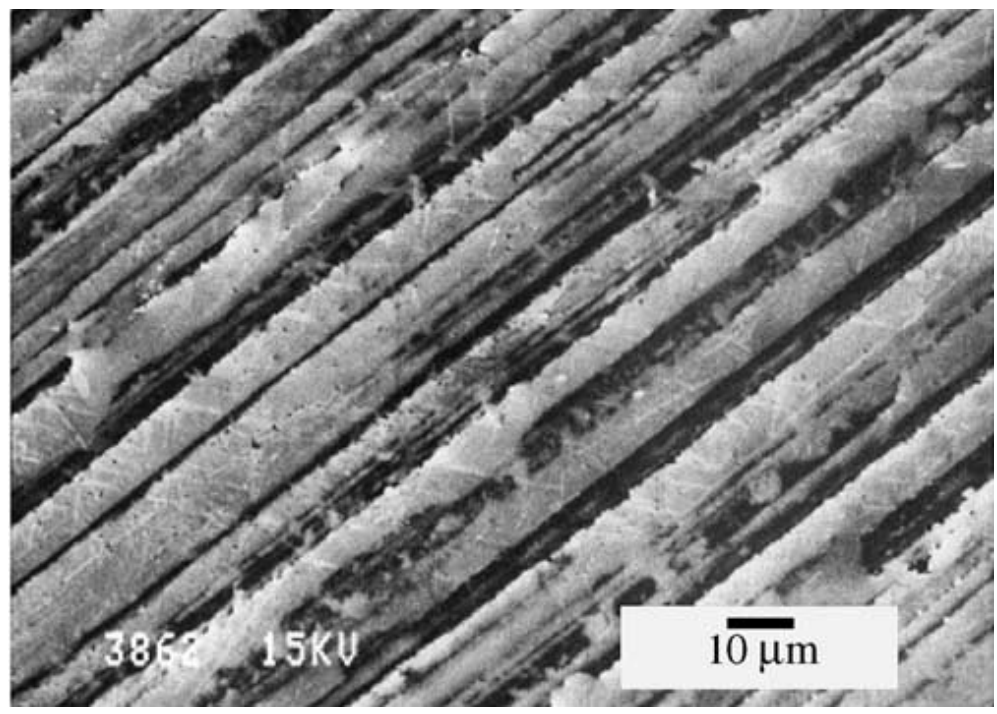

Figure 5: $\quad$ SEM results for the air-passivated specimen.

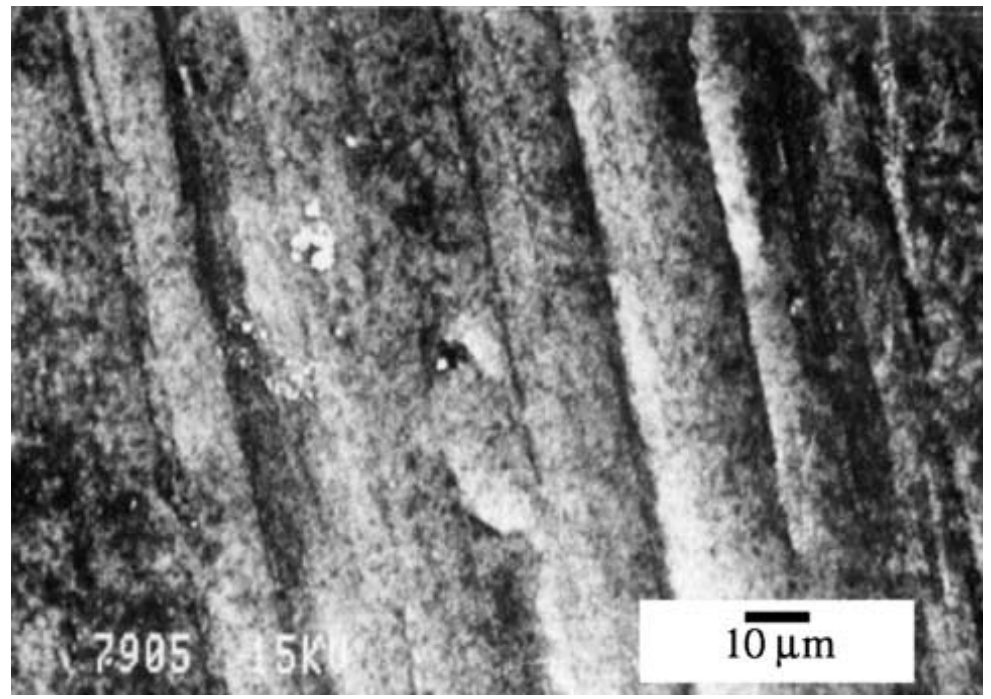

Figure 6: SEM result for the chromium-passivated specimen.

XPS peaks of $\mathrm{O}_{1 \mathrm{~s}}, \mathrm{Cr}_{2 \mathrm{p}}$, and $\mathrm{Fe}_{2 \mathrm{p}}$ in the passive film at different sputtering times were obtained.

The $\mathrm{O}_{1 \mathrm{~s}}$ peaks at $530.2 \mathrm{eV}$ corresponded to $\mathrm{O}^{2-}$ in oxide, at $531.3 \mathrm{eV}$ to $\mathrm{OH}$ in hydroxide species, and at $532.2 \mathrm{eV}$ in adsorbed water [3]. Fig.7 shows that bonding energy of the $\mathrm{O}_{1 \mathrm{~s}}$ peaks on the surface of the passive film was $531.1 \mathrm{ev}$ 
to $532.3 \mathrm{eV}$, which could correspond to $\mathrm{O}$ in the hydroxyl oxygen $\left(\mathrm{OH}^{-}\right)$and oxide oxygen $\left(\mathrm{O}^{2-}\right)$. When argon-ions sputtering was performed, the hydroxide contributions disappear but the oxide forms increase.

The $\mathrm{Cr}_{2 \mathrm{p} 3 / 2}$ peak at $574.2 \mathrm{eV}$ corresponded to the metallic state, at $576.2 \mathrm{eV}$ corresponding to the tetravalent oxide state, at $576.7 \mathrm{eV}$ corresponded to the trivalent oxide state, and at $577.2 \mathrm{eV}$ corresponding to the trivalent chromium hydroxide species [3]. Fig.8 shows that the bonding energy of $\mathrm{Cr}_{2 \mathrm{p}}$ peak on the surface was $576 \mathrm{eV}$ to $576.8 \mathrm{eV}$ which could be correspond to $\mathrm{Cr}$ in $\mathrm{Cr}^{4+}$ and $\mathrm{Cr}^{3+}$. Cr in the passive film was effective on improving the corrosion resistance [10].

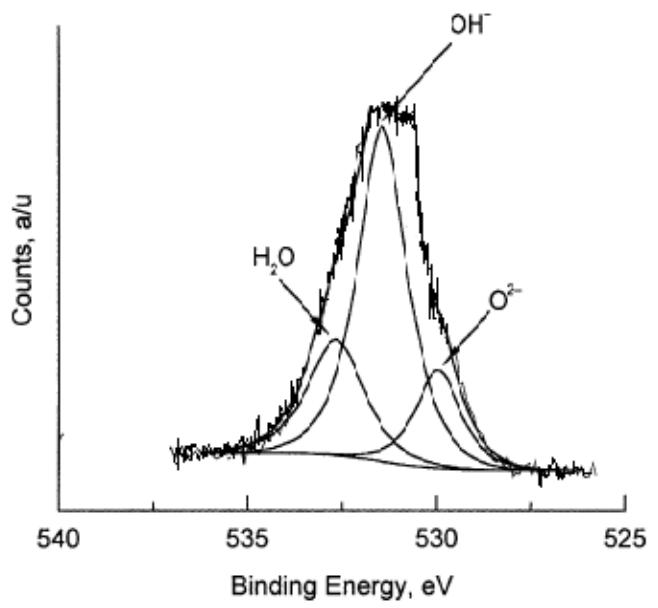

Figure 7: O1s XPS spectra for the chromium-passivated specimen.

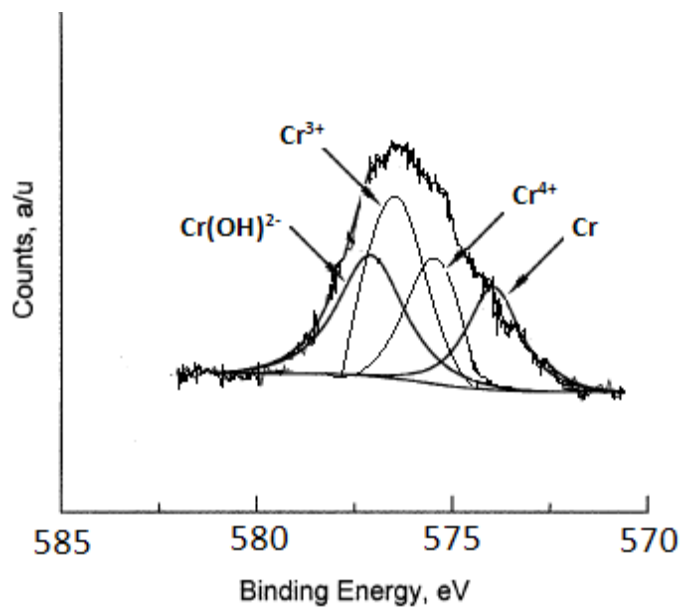

Figure 8: $\quad \mathrm{Cr}_{2 \mathrm{p}}$ XPS spectra for the chromium-passivated specimen. 


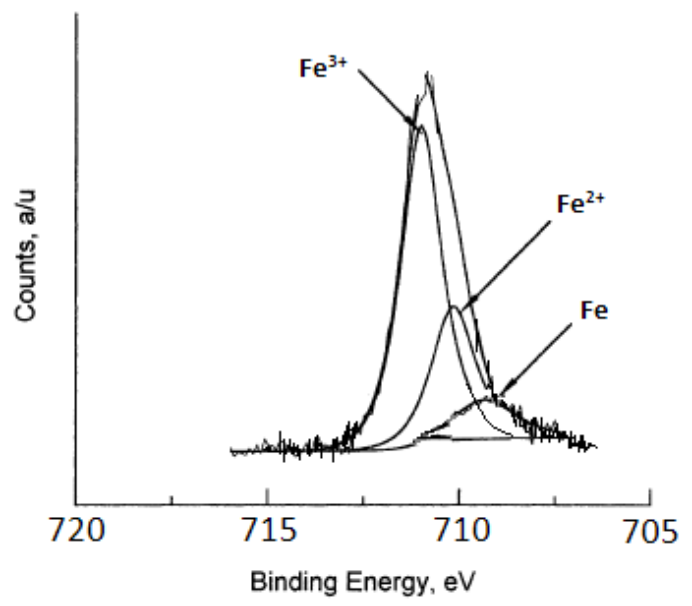

Figure 9: $\quad \mathrm{Fe}_{2 \mathrm{p}} \mathrm{XPS}$ spectra for the chromium-passivated specimen.

$\mathrm{Fe}_{2 \mathrm{P} 3 / 2}$ peak at $707.0 \mathrm{eV}$ corresponded to the metallic state, at $709.5 \mathrm{eV}$ corresponded to the divalent oxide state, and at $710.4 \mathrm{eV}$ corresponding to the trivalent oxide state. Fig.9 shows that the bonding energy of the $\mathrm{Fe}_{2 \mathrm{P} 3 / 2}$ peak on the surface was $710.1 \mathrm{eV}$ to $711.1 \mathrm{ev}$, which corresponded to $\mathrm{Fe}$ in $\mathrm{Fe}^{3+}, \mathrm{FeOOH}$, or $\mathrm{Fe}(\mathrm{OH})_{3}$.

Based on Fig.7-9, the $\mathrm{OH}$ in the outer layer of the passive film could react directly with $\mathrm{Cr}$ and $\mathrm{Fe}$ to form $\mathrm{FeOOH}$ or $\mathrm{Fe}(\mathrm{OH})_{3}$ and $\mathrm{CrOOH}$ or $\mathrm{Cr}(\mathrm{OH})_{3}$ that is stable mixture layer and could promote the repassivity of passive film [10].

The atomic concentration $\left(\mathrm{c}_{\mathrm{i}}\right)$ of the elements, as a percentage, was determined using following equation:

$$
C_{i}=\frac{A_{i} / S_{i}}{\sum\left(A_{i} / S_{i}\right)} \times 100
$$

where $A_{i}$ is the peak area and $S_{i}$ is the sensitivity factor [9]. Fig. 10 depicts the atomic concentration of each element against the argon-ion sputtering time. Sputtering time is proportional to passive layer depth [3].

It can be observed in Fig. 10 that chromium is enriched in the surface of passive layer. Oxygen and ferrous increase with the sputtering time. The passive film can be described by a bi-layer model (inner oxide and outer hydroxide layers) [3].

\section{Conclusion}

- The study presented shows the differences in corrosion behaviour of $316 \mathrm{~L}$ stainless steel after dichromate passivated. Based on this study, treating in $30 \mathrm{~g} / 1$ dichromate solution at room temperature for $72 \mathrm{hr}$ enhanced corrosion resistance significantly. 
- DC measurements shows poor corrosion resistance behaviour of airpassivated specimen: $R_{p}=1.2 \mathrm{k} \Omega \mathrm{cm}^{2}$ and $\mathrm{i}_{\text {corr }}=5.1 \mu \mathrm{A} / \mathrm{cm}^{2}$ respect to enhanced corrosion resistance behaviour of superior chromiumpassivated specimen: $R_{p}=550 \mathrm{k} \Omega \mathrm{cm}^{2}$ and $i_{\text {corr }}=0.006 \mu \mathrm{A} / \mathrm{cm}^{2}$.

- EIS results indicated passivation treatment lead to diffusion control by considering Warburg diffusion element in equivalent circuit.

- SEM examination reveals that chromium treatment is advantageous to improve smoothness.

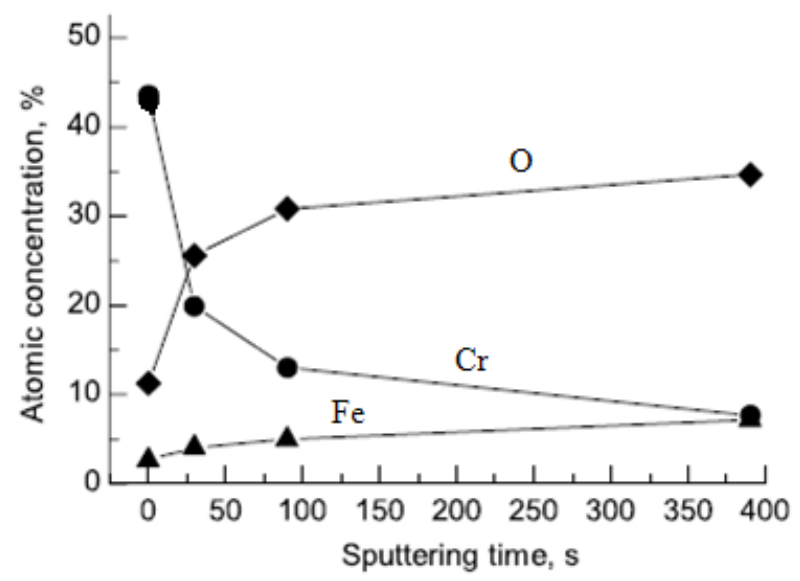

Figure 10: Atomic concentration of oxygen, chromium and ferrous elements for the chromium-passivated specimen.

The XPS results indicated that chromium-passivation treatment was advanced in the enrichment of $\mathrm{Cr}$ on the surface of stainless steel. The stable mixture compounds layer was composed of the alloy elements formed in the passive film. The $\mathrm{FeOOH}$ or $\mathrm{Fe}(\mathrm{OH})_{3}$, and $\mathrm{CrOOH}$ or $\mathrm{Cr}(\mathrm{OH})_{3}$ could exist in the outer layer of the passive film. There existed oxides of $\mathrm{Cr}$ and $\mathrm{Fe}$ in the different layer of passive film that play important roles in improving repassivation of stainless steel. The passive film can be described by a bi-layer model (inner oxide and outer hydroxide layers).

\section{Acknowledgements}

Special thanks are directed to Dr. Kermaini. This paper was supported by the Petroleum University of Technology (PUT), Pars Oil and Gas Company (POGC), and National Iranian Oil Company (NIOC).

\section{References}

[1] C.A.C. Sequeira1, D.M.F. Santos1, J.R. Sousa2,and P.S.D. Brito , The Mechanism of Oxide Film Formation on AISI 316 Stainless Steel in 
Sulphate Solution, 214th ECS Meeting, Corrosion General Poster Session, Honolulu, No. 1598

[2] Fontana, (chapter 10), Corrosion Engineering, McGraw-Hill Pub., New york, 1989

[3] Philippe Marcus, Florian Mansfeld, (eds). Analytical Methods in Corrosion Science and Engineering, CRC Press, Taylor \& Francis Group, 2006

[4] R. K. Gupta, (Chapter 10) Metal Surface Treatment and Their Chemicals, SBP Consultant and engineering PVT. Ltd, 2003

[5] ASM A967 Standard Specification for Chemical Passivation Treatments For Stainless Steel Parts, 2000

[6] Tetsuo Fujii, Haruo Baba, The Effect of Oxidizing Ion on The Passivity, Corrosion Science (31), pp. 275-280, 1990

[7] Maryam Ehteshamzade (chapter 4), Application of EIS in corrosion study, Shahid Bahonar University Kerman p. , 2006

[8] SCK-CEN, Belgian Nuclear Research Centre, Boeretang Belgium, Electrochemical impedance spectroscopy for the detection of stress corrosion cracks in aqueous corrosion systems at ambient and high temperature" Corrosion Science (47), 125-143, 2005

[9] Y. Xingwen, C. Chunan, Y. Zhiming, Z. Derui, Y. Zhongda, Study of double layer rare earth metal conversion coating on aluminium alloy LY12, Corrosion. Science(43), 1283-1294, 2001

[10] X.Y. Wang, Y.S. Wu, L. Zhang, and Z.Y. Yu, Atomic Force Microscopy and X-Ray Photoelectron Spectroscopy Study on the Passive Film for Type 316L Stainless steel, Corrosion Science, 57(6), pg 540, 2001

[11] EG\&G Princeton Applied Research, Application Note Ac-1, Basic of ac Impedance Measurements, New Jersey, 1992 